\title{
Cranio-Cervical Junction Meningioma: A Rare Case Report
}

\author{
Sukriti Das ${ }^{1 *}$, Asit Chandra Sarker ${ }^{1}$, Dipankar Ghosh ${ }^{1}$, Kanji Fatema Ishrat Zahan ${ }^{1}$, Mohammed Shamsul Islam \\ Khan $^{1}$, Akhlaque Hossain Khan ${ }^{2}$ and Bipin Chaurasia ${ }^{3}$
}

${ }^{1}$ Department of Neurosurgery, Dhaka Medical College \& Hospital, Bangladesh

${ }^{2}$ Department of Neurosurgery, Bangabandhu Sheikh Mujib Medical University, Bangladesh

${ }^{3}$ Department of Neurosurgery, Tarai Hospital and research centre, Birgunj, Nepal

*Corresponding author: Sukriti Das, Associate Professor, Department of Neurosurgery, Dhaka Medical College \& Hospital, , Dhaka, Bangladesh

\begin{abstract}
Background: Meningioma is a benign and slowly growing intradural extramedullary spinal cord tumor. It arises from the arachnoid cap cell of the meninges. Meningioma of the cranio-cervical junction is a rare and unique diagnosis of about 1.8-3.2\% of all meningiomas. These tumors usually grow slowly and develop symptoms gradually but, sometimes with sudden onset of quadriplegia. The lesions are usually very large at the time of diagnosis. Post-surgical tumor recurrence is high due to failure of complete removal of the tumor (Simpson grade-I) as critical vascular structures, brainstem and cranial nerves are close to it.

Case Report: We presented a case of a cranio-cervical junction meningioma of a 45-year-old female who presented with neck pain with headache and gradual weakness of all 4 limbs that made her unable to walk without support and bed-ridden gradually. She also developed urinary incontinence and was catheterized. On examination, the patient was conscious and alert, muscle power was of MRC grade 0/5 in all groups of muscles in all 4 limbs. All deep tendon reflexes were exaggerated in both upper and lower limbs with positive Hoffmann sign bilaterally and bilateral planter extensor response. All modalities of sensations were diminished up to $\mathrm{C}_{4}$ vertebral level. MRI of cervical spine was obtained, and the diagnosis of cranio-cervical junction meningioma came out.

Conclusion: After complete surgical removal (Simpson grade I) of the tumor, post-operative ICU management is very vital. Extubation and recovery may need long time to return to normal life. So pre-operative counseling and post-operative ICU support is mandatory to operate cranio-cervical junction meningioma.
\end{abstract}

Keywords: Cranio-Cervical Junction; Meningioma; Intradural-Extramedullary; Simpson Grade; Gross Total Removal

\section{Introduction}

Meningiomas were named by Harvey Cushing in 1922 and described as common tumors that arise from arachnoid cap cells which form the outer lining of the arachnoid membrane and from related cells such as arachnoid fibroblasts and the meningoblast [1-3]. These tumors are generally benign tumors and carry good prognosis. Meningiomas are adult onset tumors with age incidence ranging from 20-60 years and peak at around 40 years. There is also a sex preponderance with female, especially with spinal cord meningioma [4]. Cranio-cervical junction meningioma accounts for $1.8 \%-3.2 \%$ of all meningiomas [5]. These lesions are often large at the time of diagnosis [6]. Meningioma of the cranio-cervical junction is a rare and unique diagnosis among meningiomas [5-
7]. They originate from the arachnoid cap cells of the lower part of the clivus and upper aspect of C2 lamina. They are intradural and extramedullary tumor. Here, meningioma is a well circumscribed oval or round, lobulated tumor attached to the dura. This location often causes compression of the spinal cord with slowly progressive features of myelopathy [4]. Mortality, morbidity and recurrence are $16.7 \%, 5.5 \%$ and $5.5 \%$ respectively [8]. Approximately $70 \%$ of all tumors in cranio-cervical junction are meningiomas [5]. Complete resection should be the primary goal of surgery but, is often difficult to achieve due to their close relationship with critical vascular structures, the brainstem and cranial nerves. Therefore, Simpson grade-I resection is rarely achieved which might explain the higher risk of recurrence $[1,2,7,9]$. 


\section{Case Report}

A 45-year-old lady presented with neck pain and weakness of all four limbs. Weakness was gradual in onset, progressive in nature and associated with stiffness. Weakness started from right upper limb, then gradually involved right lower limb followed by left upper limb and lastly left lower limb. She developed grip weakness of her right hand at the beginning, then she noticed herself limping also developed inability to perform household works, difficulty in walking without support and ultimately became bedridden. She also developed urinary incontinence and constipation in the meantime. She complained neck pain for about last 3 years which was episodic, located in back and side of the neck and lower part of back of the head being constant dull aching in nature, mild to moderate in intensity, non-radiating, with no diurnal variation, no known aggravating factors and usually relieved by pain killer [10].

On examination- the patient was conscious and alert, but ill looking and bed ridden. Motor examination of all four limbs revealed- bilateral symmetrically reduced muscle bulk, increased muscle tone. Muscle power was of MRC grade-0/5 in all groups of muscle in all four limbs, all deep tendon reflexes of upper and lower limbs were exaggerated with bilateral planter extensor and bilateral positive Hofmann sign. Clinical diagnosis was made as a case of spastic quadriplegia due to compressive cervical myelopathy (High cervical).

MRI of cervical spine with and without contrast revealed a welldefined lobulated mass measuring about $56.3 \mathrm{~mm}$ x17.4 $\mathrm{mm}$ that is intermediate in intensity in both $\mathrm{T}_{1} \mathrm{Wl} \& \mathrm{~T}_{2} \mathrm{Wl}$, is seen at craniocervical junction \& upper cervical canal causing compression at lower pons, upper cervical cord and also over corresponding $\mathrm{C}_{2}$ nerve roots. After IV contrast-Contrast enhancement of the lesion is seen. X-ray of cervical spine was normal.

The patient was diagnosed as a case of spastic quadriplegia due to cranio-cervical junction meningioma. She underwent midline sub-occipital craniectomy with laminectomy at $\mathrm{C}_{1}$ and $\mathrm{C}_{2}$ with complete surgical excision of tumor (Simpson grade-I). Histopathology report also revealed meningioma (WHO grade-I). Post-operative follow-up was given in ICU and on the $10^{\text {th }}$ POD the patient was extubated with motor power of MRC grade 3/5 as she was shifted to general ward. She was discharged on the $15^{\text {th }}$ POD. Now, after 2 months following operation, she is able to walk with support having motor power of MRC grade $4+/ 5$ in all four limbs (Figures 1-8).
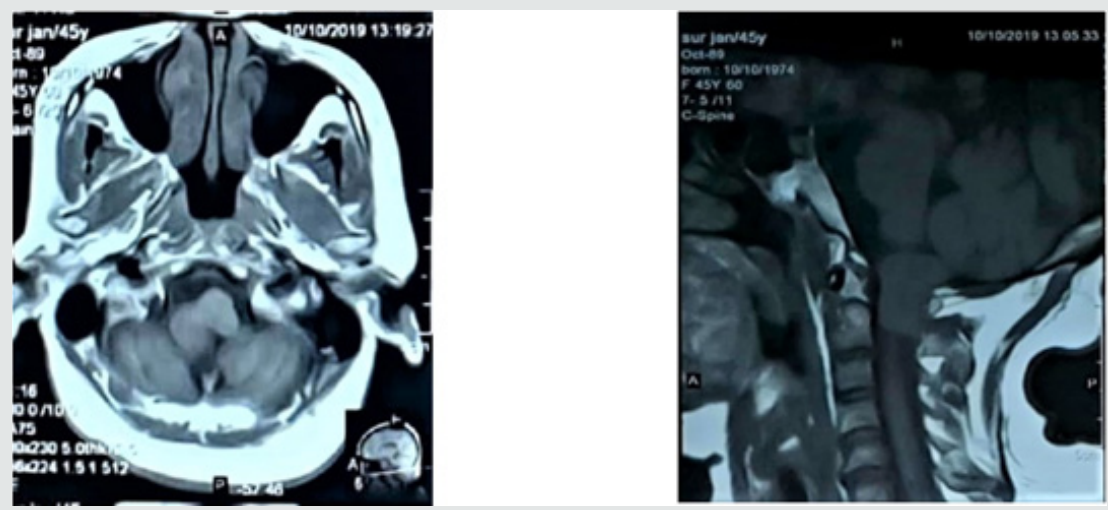

Figure 1: $\mathrm{T}_{1} \mathrm{WI}$ axial and sagittal film showing tumor at cranio-cervical junction.
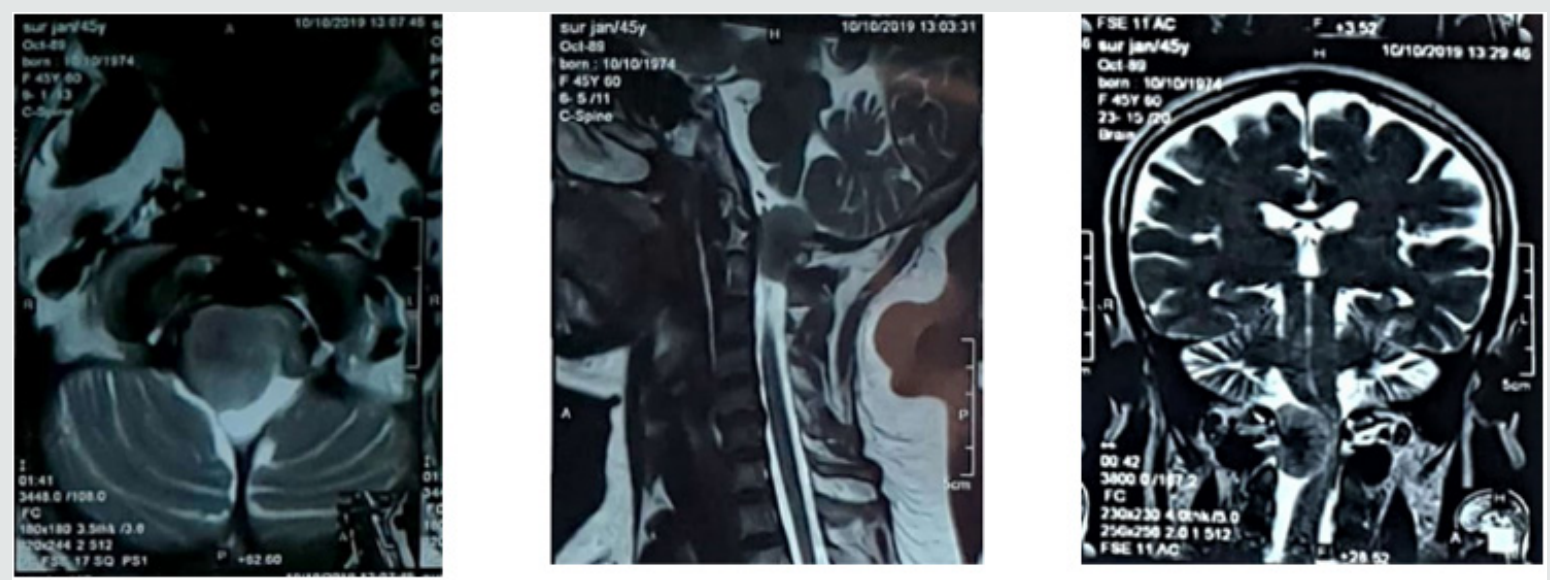

Figure 2: $\mathrm{T}_{2} \mathrm{WI}$ axial, sagittal and coronal film showing tumor at cranio-cervical junction. 

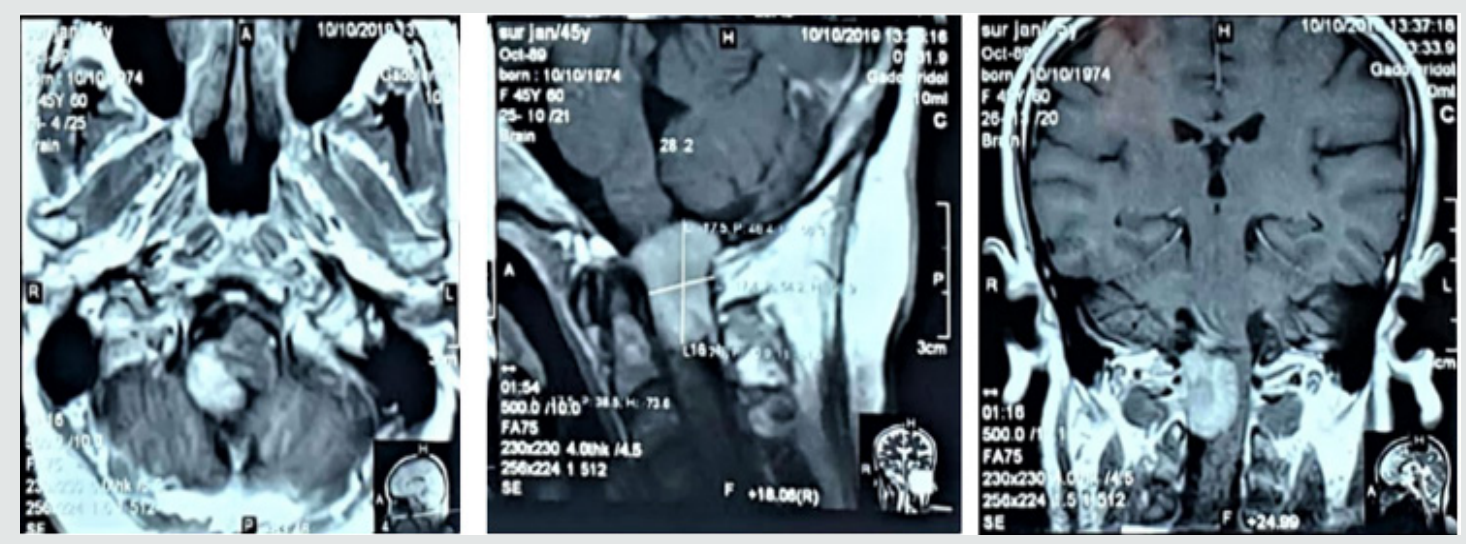

Figure 3: $\mathrm{T}_{1} \mathrm{WI}$ post-contrast axial, sagittal and coronal film showing contrast uptake by the tumor.
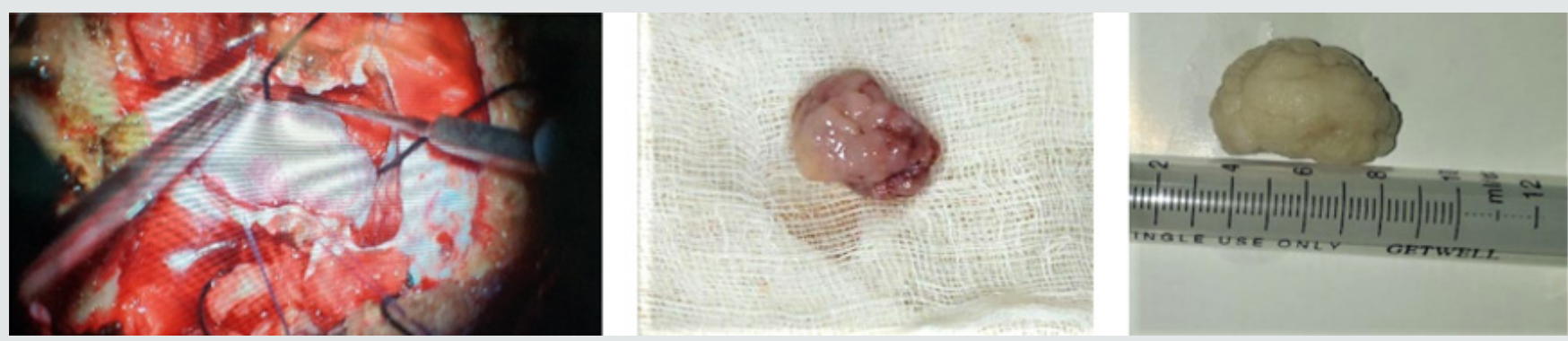

Figure 4: Per-operative view of the tumor and after complete excision.

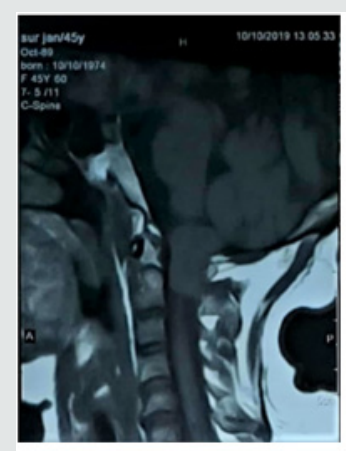

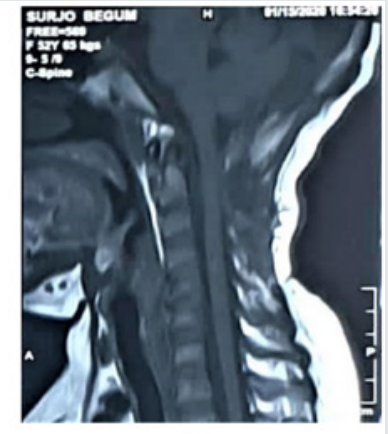

b

Figure 5: 'a' pre op $T_{1}$ WI sagittal film showing tumor and 'b' post op $T_{1}$ WI sagittal film showing complete removal of tumor.

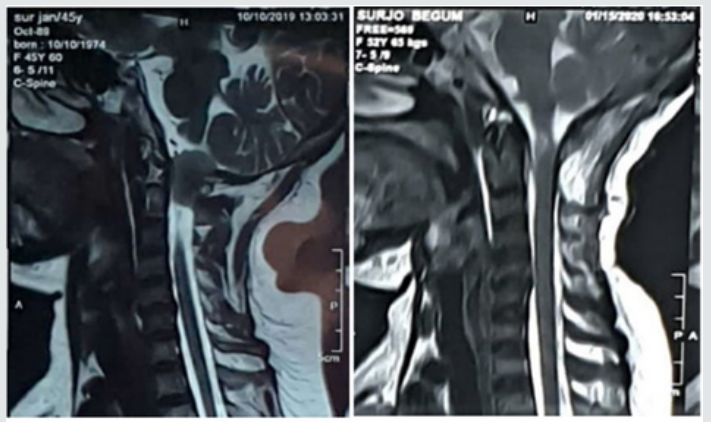

Figure 6: 'a' pre op $\mathrm{T}_{2}$ WI sagittal film showing tumor and 'b' post op $\mathrm{T}_{2} \mathrm{WI}$ sagittal film showing complete removal of tumor. 


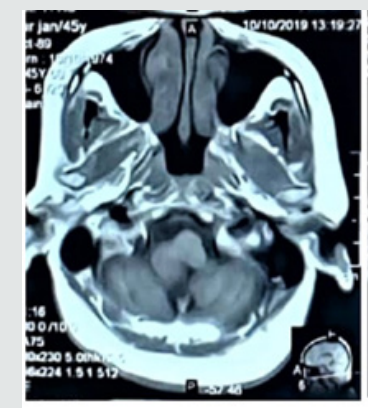

a

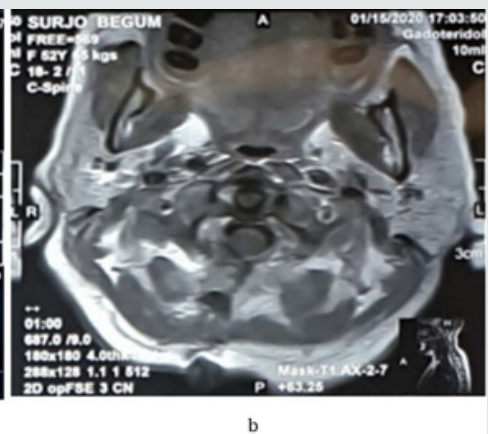

Figure 7: 'a' pre op $\mathrm{T}_{1} \mathrm{WI}$ axial film showing tumor and ' $b$ ' post op $T_{1} W I$ axial film showing complete removal of tumor.

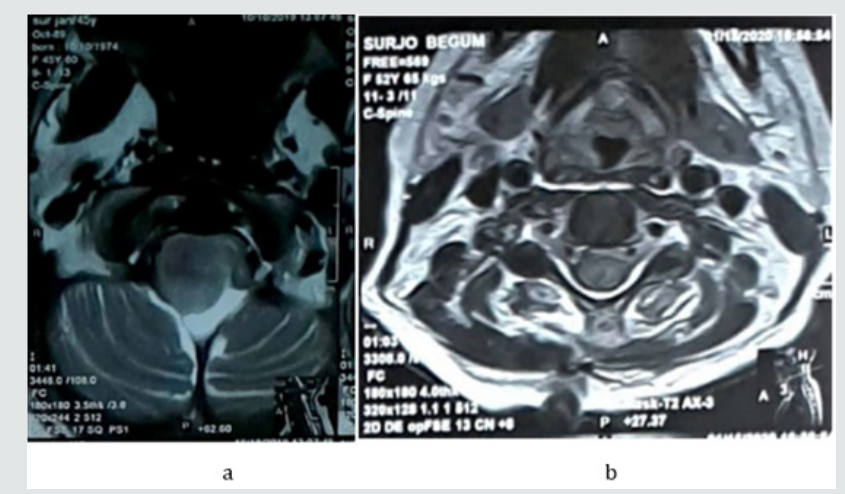

Figure 8: ' $a$ ' pre op $\mathrm{T}_{2} \mathrm{WI}$ axial film showing tumor and ' $b$ ' post op $T_{2} W I$ axial film showing complete removal of tumor.

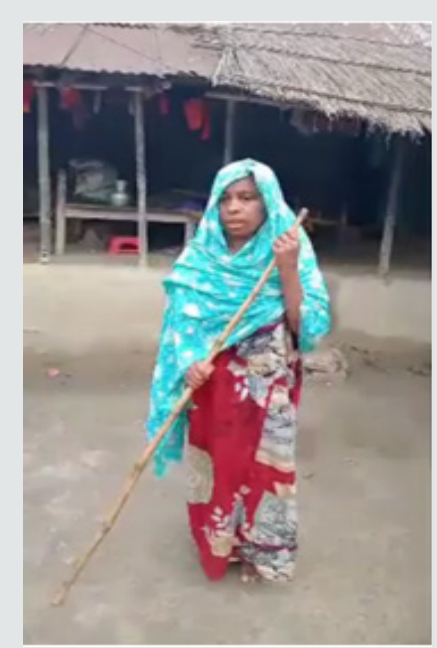

Figure 9: The patient is walking after 50 days of surgery.

\section{Discussion}

We presented our experience in managing cranio-cervical meningioma and its outcome as such tumors are rare (1.8 to $3.2 \%$ of all meningiomas) [1]. It is usually benign intradural extramedullary tumor arising from arachnoid cap cells of outer arachnoid layer.
Based on the origin of the tumor matrix or dural attachment, these lesions are of two types-

(i) cranio-spinal and

(ii) spino-cranial.

They are different in their origin, anatomical location and relation with neighboring structures. Spino-cranial tumors are associated with higher rates of total resection with fewer postoperative complications like cranial nerve deficits and vascular damage as mean tumor volume are smaller than cranio-spinal variety. These tumors impinge on the spinal cord earlier than their cranial counterparts, so they cause symptoms at a smaller size resulting in earlier diagnosis. They are about $30 \%$ of all craniocervical junction tumor and having higher rate of total resection whereas cranio-spinal meningiomas are relatively asymptomatic with often delayed symptoms and become large at the time of diagnosis along with invasion of surrounding neuro-vascular structures. In these cases, total resection becomes difficult and surgical removal sometimes invite complications. Vertebral artery encasement and cranial nerve deficits are two very vital factors that have to be considered before planning surgery.

Cranio-cervical junction meningiomas are diagnosed by CT scan and MRI of brain with CTA and MR angiogram being sometimes in need. Tumor may be anterior to the neuroaxis- that is anterior or antero-lateral tumors are located ventral to the dentate ligament while posterior and postero-lateral tumors are dorsal to the dentate ligament. The extent of tumor resection was complete or sub-total, but our target was the complete excision of the tumor (Simpson grade-I). As it is a benign tumor, its recurrence rate is scanty. Total resection of cranio-cervical junction meningioma in previous studies was achieved typically in $61-100 \%$ of cases. When the tumor engulfs critical neuro-vascular structures sometimes it is treated conservatively. Sometimes radiotherapy is used in conjunction with surgery, but result is not good. In our patient, posteriorly placed cranio-spinal tumor was big and presented with quadriplegia without cranial nerve palsy or vascular encasement. We planned for posterior midline suboccipital craniectomy with laminectomy of $\mathrm{C}_{1}$ and $\mathrm{C}_{2}$. After durotomy, the whole big tumor was revealed, and excision of the tumor was done piece by piece.

Gross total resection was achieved in $45 \%$ of patients and sub-total in $55 \%$ of cases. But, in our case we performed complete resection (Simpson grade-I) of the tumor. The most common postoperative complications were dysphagia, palsy of cranial nerve- IX, $\mathrm{X}, \mathrm{XI}, \mathrm{XII}$. But, in our case the patient having no visible cranial nerve palsy, but she had delayed extubation. We obtained an MRI at 15 days after surgery to detect any residual tumor or any collection or pseudo meningocele or CSF leak. The patient was advised to have MRI scan at 2, 6 and 12 months after surgery. 
Our patient improved gradually regarding neck pain, hypoesthesia, paresthesia and sphincter disturbance at 2 around months around following surgery and became able to walk with support. NO evidence of any cranial nerve deficit, CSF leak or cerebellar infarction was seen.

\section{Conclusion}

Surgical management of cranio-cervical junction meningioma is a challenge to neurosurgeons especially in case of tumors involving neighboring neurovascular structures. Our case had quadriplegia with autonomic disturbances but no vertebral artery encasement or cranial nerve palsy. Though cranio-spinal tumor presents lately and becomes large in size at the time of diagnosis and usually involves surrounding neurovascular elements, it is very difficult to perform complete resection with high rate of post-operative complications. We are happy to say that in our case, we performed complete resection of the tumor without any neurovascular complication. Functional outcome was good (the patient became able to walk with support at 2 months following surgery) with significant improvement of sphincter dysfunction and sensory symptoms.

\section{References}

1. Cushing H and Eisenhardt L (1939) Meningiomas: Their Classification, Regional Behavior, Life History and Surgical End Results. Arch NeurPsych 41(3): 657-658.
2. Alam S, Chaurasia BK, Shalike N, Uddin AN, Chowdhury D, et al. (2018) Surgical management of clinoidal meningiomas: 10 cases analysis. Neuroimmunol Neuroinflammation 5: 21.

3. Moududul Haque, Nwoshin Jahan, Shahnawaz Bari, KM Tarekul Islam, Shamsul Alam, et al. (2019) Two cases of atypical foramen magnum meningioma presenting as rotatory paralysis. J Neurol Stroke 9(4): 180182.

4. Wilkins RH and Rengachary SS (1985) Neurosurgery. Vol 1, Section D. McGraw Hill, New York Pp: 614.

5. Arnautovic KI, Al-Mefty O, Husain M (2000)Ventral foramen magnum meningiomas. J Neurosurg 92: 71-80.

6. Boulton MR, Cusimano MD (2003) Foramen magnum meningiomas: concepts, classifications, and nuances. Neurosurg Focus 14(6): e10.

7. Yasuoka S, Okazaki H, Daube JR, MacCarty CS (1978) Foramen magnum tumors. Analysis of 57 cases of benign extramedullary tumors. J Neurosurg 49(6): 828-838.

8. Dührsen L, Emami P, Matschke J, Abboud T, Westphal M, et al. (2016) Meningiomas of the Craniocervical Junction - A Distinctive Subgroup of Meningiomas. PLoS ONE 11(4): e0153405.

9. Bassiouni H, Ntoukas V, Asgari S, Sandalcioglu EI, Stolke D, e al. (2006) Foramen magnum meningiomas: clinical outcome after microsurgical resection via a posterolateral suboccipital retrocondylar approach. Neurosurgery 59(6): 1177-1185.

10. Hwang WL, Marciscano AE, Niemierko A, Kim DW, Stemmer-Rachaminov AO, et al. (2016) Imaging and extent of surgical resection predict risk of meningioma recurrence better than WHO histopathological grade. Neuro oncology 18(6): 863-872.

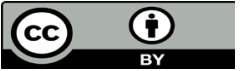

This work is licensed under Creative Commons Attribution 4.0 License

To Submit Your Article Click Here:

Submit Article

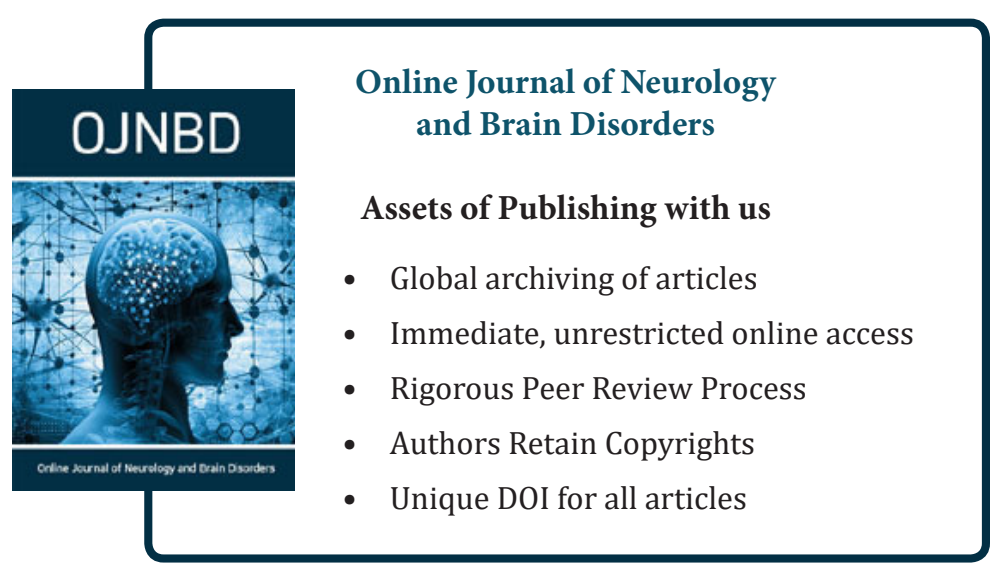

